\title{
Preface
}

\section{First Steps in Addressing \\ Health Disparities in Thoracic \\ Surgery}

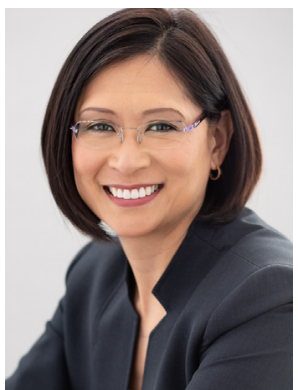

Cherie P. Erkmen, MD

Editor

In the constitution of the World Health Organization, health is defined as "one of the fundamental rights of every human being without distinction of race, religion, political belief, economic or social condition."1 The COVID-19 worldwide pandemic demonstrated that health is very much a function of all these factors. We can empirically measure differences in health outcomes and mortality. However, to address root causes and develop interventions to correct health disparities, we must delve deeper into mechanisms. We must scrutinize biased assumptions, discriminatory actions, and a failure to act.

Firmly held traditions may impede our path to health equity. For example, we in the thoracic surgery community widely accept race correction of spirometry results. Race correction originated from scholarly work of Thomas Jefferson and a contemporary physician named Samuel Cartwright. They asserted innate, biological differences between races, specifically, pulmonary "deficiency in the Negro" of "20 percent." Furthermore, they used these assumptions to justify slavery by concluding that forced labor invigorates "the red vital blood sent to the brain that liberates their minds when under the white man's control, and it is the want of sufficiency of red vital blood that chains their minds to ignorance and barbarism when in freedom."2,3 In present-day evaluations, spirometry software automatically invokes a correction of $10 \%$ decrease for black patients and $6 \%$ decrease for Asian patients ${ }^{4}$ once race is entered. ${ }^{5}$ If we pause to think about the implications of this, we assume that non-white patients have less "vital capacity" than white patients. If we assume race impacts pulmonary function, how do we consider environmental, social, economic, educational, employment, and other factors in this calculation? Or, are we using race as an oversimplified proxy for all these factors? From a utilitarian standpoint, how should we correct for people of mixed race or races other than black and white? Is race a biologic difference or social construct? Are disparate outcomes a biologic difference and/or a result of access to quality care? 
As overwhelming as these questions are, our focus should be on achieving health equity. Health equity does not mean that everybody receives the same treatment, nor does it mean that everyone is expected to have the same outcome. Health equity is striving to reach each individual's highest potential of health. In this landmark work, we begin to develop a framework for answering these questions of health disparity within thoracic surgery. We look at the historical context of health policy and health care delivery in the United States (articles by Magarinos and colleagues and Nadeem and Kaiser), health disparities with specific disease process within our practice (articles by Raman and colleagues, Elliott and colleagues, Espinosa and Raja, Randhawa and colleagues, and Pickens), how study design, enrollment, and data management impact our approach to health disparity (articles by Adnan and colleagues, Charles and colleagues, and Mitchell and colleagues), and how disparities in thoracic training and workforce impact health disparities (Ceppa and Godoy and colleagues).

The past few years have prompted many of us to see structural racism, sexism, and discrimination. Culturing a curiosity allows us evaluate problems objectively, to "think more deeply and rationally." Diversive curiosity, or "curiosity associated with the interest in exploring unfamiliar topics and learning something new," is associated with quality problem-solving and originality. ${ }^{7,8}$ Understanding and addressing health disparities in our practice demand this first step of curiosity. We should try to avoid divisive shame or blame, but instead question mechanistic underpinnings of health disparity. Unfortunately, we have seen a backlash against exploration of structural discrimination. It has been disparaged as "woke" or "cancel" culture. Being curious is not a political stance; it is the foundation of empathy and innovation, which is desperately needed in the field of health disparities.

This issue represents innovation from the contributors, who have analyzed current evidence with a fresh perspective. I would like to thank the authors, who have exceeded all expectations to deliver something new to thoracic surgery. I am indebted to John Vassallo for his enthusiastic support, and the staff at Elsevier, especially Jessica Cañaberal, who has been a skilled and consummate professional. I am most grateful to Virginia Litle for having the courage and vision to conceive a health disparities issue of Thoracic Surgery Clinics. It has been one of the greatest honors of my career to be entrusted with this meaningful endeavor at this critical time.

Cherie P. Erkmen, MD Temple University Health Systems Temple University Hospital-Thoracic Surgery 3401 North Broad Street Suite C-105, Parkinson Pavilion Philadelphia, PA 19140, USA

E-mail address: cherie.p.erkmen@gmail.com

\section{REFERENCES}

1. Available at: https://www.who.int/about/governance/ constitution. Accessed.

2. Cartwright S. Report on the diseases and physical peculiarities of the Negro race. New Orleans Med Surg J 1851;7:691-715.

3. Cartwright S. Slavery in the light of ethnology. In: Elliott $\mathrm{E}$, editor. Cotton is king and proslavery arguments. Augusta, GA: Abbott \& Loomis; 1860.

4. Lujan HL, DiCarlo SE. Science reflects history as society influences science: brief history of "race," "race correction," and the spirometer. Adv Physiol Educ 2018;42(2):163-5 PMID: 29616572.

5. Braun L. Race, ethnicity and lung function: a brief history. Can J Respir Ther 2015;51(4):99-101.

6. Available at: https://hbr.org/2018/09/the-businesscase-for-curiosity. Accessed.

7. Hardy JH III, Ness AM, Mecca J. Outside the box: epistemic curiosity as a predictor of creative problem solving and creative performance. Pers Individ Diff 2017;104:230-7.

8. Available at: https://today.oregonstate.edu/archives/ 2016/nov/curiosity-can-predict-employees\%E2\%80\% 99-ability-creatively-solve-problems-research-shows. Accessed. 